1069 THE UNPRESCRIBED USE OF ANTIBIOTICS IN CHILDREN ADMITTED TO HOSPITAL. Malliswari Challapalli and Daniel G. Cunningham (Spon. by George F. Smith) Mercy Hospital, Chicago, Department of Pediatrics We have previously reported that approximately $7 \%$ of childrer who present for medical care with common respiratory illnesses are already being treated with unprescribed antibiotics. The are already being treated with unprescribed antibiotics. The purpose of the present study is to determine the incidence of
antibiotic abuse(self-medication with unprescribed antibiotics) antibiotic abuse (self-medication with unprescribed antibiotics) in children with more serious febrile illnesses. It is anticipated that we will have 400 patients by $4 / 85$. To date we have from 104 febrile patients. 8 of 104 (7.69\%) children were found to have antibacterial activity in the urine as determined by bioassay. Of the 8 positive specimens only 2 were from children whose care takers admitted to antibiotic abuse. One received exythromycin and the other penicillin. Sources of antibiotic were sibling's erythromycin and amoxicillin from a previous illness. Data related to 1) duration of illness, 2) patient age, 3) symptom categories (URI, Pneumonia, Asthma, Gastroenteritis, Sepsis, Fever), 4) similar illness in the past, 5) presence of siblings, 6) primary care taker's marital status, 7) abuse was significantly higher in the asthmatics $(P \quad .01)$ and no correlation was demonstrated to incidence of abuse and other mentioned variables. We conclude that antibiotic abuse is a significant problem and questioning was not a reliable abuse direction technique. The incidence of abuse in this study is similar to previously reported incidence in outpatients fxom a different geographical area.

\section{$\dagger 1070$}

BUCCAL CELLULITIS (BC) RE-EVALUATED. Stephen A.

Chartrand and Christopher J. Harrison. (Spon. by

Dept. of Pediatrics C. Boerth). Univ. of Oklahoma Medical School, College of Medicine, Dept. of Pediatrics, Mobile.

Previous studies of $B C$ have reported conflicting data on the incidence of co-existent bacteremia and meningitis. Otitis media $(O M)$ is reportedly found in $75 \%$ of patients and, when unilateral, is said to always occur on the ipsilateral side (suggesting lymphangitic spread to the cheek). To more accurately define the clinical picture of $\mathrm{BC}$, we reviewed the records of 55 children with a clinical diagnosis of primary (no overlying skin trauna) BC. There were 32 males and 23 females with a median age of 11 (range 1.5-64) months. OM was present in 22 (40\%) patients. The contralateral ar was involved in 5 of 13 patients with unilateral OM. Blood cultures were positive in 27 (53\%) of 51 patients and grew $\mathrm{H}$. influenzae $\mathrm{b}$ (HIB) in $25(92.5 \%)$. Two infants with $\frac{\mathrm{s}}{\mathrm{d}}$. pneumoniae and $\underline{\mathrm{s}}$. $\frac{\text { agalactiae infections were clinically in- }}{\text { the remaining patients. Cellulitis cultures }}$ were positive in $6(17 \%)$ of 35 attempts. Cerebrospinal fluid (CSF) cultures were positive in $3(9.4 \%)$ of 32 patients; one additional child whose CSF was not initially examined later developed meningitis. These data suggest that: (a) bacteremia is commonly present in $\mathrm{BC}$, (b) the pathogenesis of $\mathrm{BC}$ does not us ally involve lymphangitic extension from the middle ear, (c) lumbar punctures should always be performed because of the associated risk of meningitis and (d) BC is predominately but not exclusively due to HIB. Initial therapy should include parenteral antimicrobial coverage for HIB and Gram positive organisms.

VARICELLA (VAR) INFECTION IN DAYCARE CENTERS (DCC).

$1071 \frac{\text { Stephen }}{\text { by Robert }} \frac{\text { Chartrand and Wendy }}{\text { C. Boerth). Mniv. Of }}$. Mouth Alabama College of Medicine, Department of Pediatrics, Mobile.

There are no published data on the epidemiology or economic consequences of VAR infections in DCC. We investigated the VAR attack rate and financial burden due to VAR infection on families of children attending DCC. We administered a retrospective questionnaire to parents of children attending four of the largest DCC in Mobile during the winter/spring of 1983/84. At that time VAR was epidemiring the winter/spring of $1983 / 84$. An the showed that $71(42 \%)$ of 171 reportedly susceptible children developed clinical disease. The mean (range) number of days lost per child from the DCC was $5.4(2-14)$. Forty-one $(78 \%)$ of the 55 families affected lost a mean (range) of $3.9(0-15)$ days of work. This included a total of 90 days of sick leave, 49 days off without pay, 18 days off with pay and 9 vacation days. Sixoff without pay, teen (29\%) of the families lost a mean (range) of $\$ 146.00$ ( $\$ 35-$ $400.00)$ in wages, not including ongoing DCC enrollment fees. Additional medical bills/babysitting costs for 41 children totaled $\$ 1258.00$ (mean $=\$ 31.00 /$ child). These data suggest that VAR infections in DCC have a significant financial impact on families. Immunization of susceptible DCC enrollees with live attenuated varicella vaccine, if found to be safe in the daycare setting, may prevent the spread of infection and reduce the financial strain on families.
1072 STAPHYLOCOCCUS EPIDERMIDIS (S. EPI.) SEPSIS 1072 Chawla, M.Curtis-Cohen. Dept. Pediatrics, S.epi is known to cause infection in neonates with indwelling catheters or ventricular shunts. Recent1y it has been associated with infections in infants with no such foreign bodies. In our ICN from July through no such foreign bodies. In our ICN from July through October S.epi was the most common cause of sepsis, re-
sponsible for $6 / 10$ cases. BW and gest. age in these 6 infants was $1425 \pm 434$ grams and $31 \pm 3$ weeks (mean $+S D$ ). A11 infants had umbilical venous (1) or arterial (5) catheters which were removed $7-45$ days prior to onset of symptoms. 4 infants had peripheral venous catheters in place. Onset of symptoms occurred between 11 and 54 days of age. In all infants symptoms were indolent and present for $>24$ hours prior to evaluation for sepsis. They included: tachypnea and hypoxemia (2), apnea and bradycardia( 3 ), feeding intolerance(3), lethargy (5), and fever ( 1$)$. 3 infants were started on ampicillin and gentamicin with no improvement, 2 were begun on vancomycin, and 1 was untreated prior to 2 sets of positive blood cultures. S.epi sensitive to vancomycin and chloramphenicol was isolated from the blood of all 6 infants. After 24 hours of vancomycin all improved dramaticaily. A11 urine and CSF cultures were sterile.

Although S.epi frequently contaminates cultures, the organism must be regarded as a potential pathogen in the ICN and treated aggressively.

91073 INTRAVENOUS HUMAN IMMUNE GLOBULIN PREVENTS NEUTROPENIA, NEUTROPHIL SUPPLY EXHAUSTION AND DEATH IN EXPERIMENTAL GROUP B STREPTOCOCCAL SEPSIS. RD Christensen, HR Hill, G Rothstein, TE Harper, U of Utah, SLC, UT. In neonatal animals with group $B$ streptococcal (GRS) sepsis, neutropenia and depletion of the marrow neutrophit (neut) reserves are prevented and survival markedly improved by murine hybridoma anti-GBS antibody. This product is not practical for use in human infants, however, because of its non-human origin. Therefore, we tested intravenous immune globul in (IVIG) for its effect on blood and marrow neut kinetics and survival. Groups of 15-30 neonatal rats were transthoracically inoculated with $10^{5}$ GBS/gm. All animals which received $40 \mathrm{ml} / \mathrm{kg}$ (containing $5 \mathrm{gm} \%$ $\mathrm{IgG}$ ) lived (albumin control survival $=0 \%$ ), 95\% given $20 \mathrm{ml} / \mathrm{kg}$
survived, and $20 \%$ given $2 \mathrm{ml} / \mathrm{kg}$ lived. Even when delayed for $2 \mathrm{~h}$, $20 \mathrm{ml} / \mathrm{kg}$ resulted in $100 \%$ survival. Survival fell to $57 \%$ when IVIG was delayed for $6 \mathrm{~h}$ and to $46 \%$ at $22 \mathrm{~h}$. IVIG also facilitated IVIG was delayed for $6 \mathrm{~h}$ and to $46 \%$ at 2 . the neut inflammatory response: 1) IVIG recipients released ned more promptly from the marrow (after $2 \mathrm{~h}$, IVIG animals had re-
leased $5.5 \pm 0.8 \times 10^{6}$ neut from reserves vs $1.2 \pm 0.6 \times 10^{6}$ in controls, $x \pm S E M, p<0.05), 2)$ IVIG prevented neutropenia $\left(2763 \pm 216 / \mathrm{mm}^{3}\right.$ IVIG vs $148 \pm 38 / \mathrm{mm}^{3}$ control, $\left.\mathrm{p}<0.001\right)$, 3) neut supply did not become exhausted in IVIG recipients $\left(4.5 \pm 0.5 \times 10^{6}\right.$ reserve neut $20 \mathrm{~h}$ after inoculation vs $0.3 \pm 0.1 \times 10^{6}$ in controls, $\left.p<0.001\right), 4$ ) accumulation of neut at the site of GBS inoculation was more rapid (myeloperoxidase-a neut marker-in lung $2 \mathrm{~h}$ after inoculation $=1.7 \pm 0.2$ units
vs $1.0 \pm 0.1$ in controls, $p<0.05$ ). Therefore, IVIG markedly facilitated the neut response and improved survival. These findings provide support for a trial of IVIG in infected human neonates.
- 1074 SAFETY AND IMMUNOGENICITY OF A LIVE ATTENUATED RHESUS MONKEY ROTAVIRUS VACCINE. Cynthia Christy, John J. Kapikian, RaphaeT Dol in (Spon. by Carol ine B. Hal1). University of Rochester School of Medicine, Departments of Medicine \& Pediatrics, Rochester, NY and the National Institutes of Health, Bethesda, MD Rochester is a the worldwide cause of acute gastroenteritis Rotavirus is a major worlawide cause rhesus monkey rotavirus (RRV-1) which shares neutralization specificity with human serotype 3 , as a potential vaccine candidate. We administered RRV(106 TCID 50$)$ or placebo orally to 40 adult volunteers who had low or undetectable levels of serum neutralizing antibody under signs and symptoms of gastroenteritis, and stools were examined for RRV-1 by ELISA and tissue culture. Administration of RRV-1 did not induce iliness. RRV-1 was shed in the stools of 11/27 (53\%) vaccinees for 1 to 6 days without transmission of RRV-1 to (53\%) vaccinees for to 6 days without transmi shed wild-type other subjects, although one placebo recipient shed wild-typ rotavirus from a community-acquired infection. Fourfold or greater serum neutralizing antibody rises to RRV- 1 were noted in
a11 vaccinees $(21 / 21)$ and in $1 / 18$ placebo recipients. Serum antibody rises were also demonstrated by immune adherence hemagglutination in 20/21 volunteers who received RRV-1 and in $1 / 18$ who received placebo, and by complement fixation in $17 / 21$ who received RRV -1 and $0 / 17$ who received placebo. Transient asymptomatic serum ALT and AST rises were seen with similar frequencies in RRV-1 (3/21) and placebo (5/19) recipients. Thus, RRV-1 appears to be attenuated and highly immunos
and warrants additional study in children. 